\title{
Solving parabolic equations on the unit sphere via Laplace transforms and radial basis functions
}

\author{
Q. T. Le Gia and William McLean* \\ June 13, 2018
}

\begin{abstract}
We propose a method to construct numerical solutions of parabolic equations on the unit sphere. The time discretization uses Laplace transforms and quadrature. The spatial approximation of the solution employs radial basis functions restricted to the sphere. The method allows us to construct high accuracy numerical solutions in parallel. We establish $\mathrm{L}_{2}$ error estimates for smooth and nonsmooth initial data, and describe some numerical experiments.
\end{abstract}

Keywords: parabolic equations, Laplace transforms, unit sphere, radial basis functions

AMS subject classifications: 35R01, 65N30

\section{Introduction}

We consider the initial-value problem

$$
\partial_{t} u+A u=f(t), \quad \text { for } t>0, \quad \text { with } u(0)=u_{0},
$$

*School of Mathematics and Statistics, University of New South Wales, Sydney, Australia. Email:qlegia@unsw.edu.au, w.mclean@unsw.edu.au 
where $\partial_{t}=\partial / \partial t$ and $A$ is a linear, self-adjoint, positive-semidefinite, secondorder elliptic partial differential operator on the unit sphere. In our standard example, $-A$ is the Laplace-Beltrami operator. The source term $f(t)$ may depend on the spatial variables but we suppress this dependence in our notation, viewing $f(t)$ as an element of a function space on the sphere.

Instead of using time stepping for the numerical solution, as was done previously [4], our approach is to represent the solution of (1.1) as an inverse Laplace transform, which is then approximated by quadrature. Developed first for parabolic problems by Sheen, Sloan and Thomée [11, such an approach is also effective for some evolution equations with memory [5]. These and related papers have discussed thoroughly the time discretization, but for the space discretization have considered only piecewise linear finite elements on a bounded domain in $\mathbb{R}^{n}$. Here, we propose instead a space discretization using spherical radial basis functions (SRBFs), which are convenient for parabolic problems on Riemannian surfaces such as the unit sphere $\mathbb{S}^{n}=\left\{x \in \mathbb{R}^{n+1}:|x|=1\right\}$.

Denoting the Laplace transform of $u$ with respect to $t$ by

$$
\hat{u}(z)=\mathcal{L}\{u(t)\}:=\int_{0}^{\infty} e^{-z t} u(t) d t,
$$

we find that the solution of (1.1) formally satisfies

$$
(z I+A) \hat{u}(z)=g(z):=u_{0}+\hat{f}(z),
$$

where I denotes the identity operator. The spectrum of $A$ is a subset of the half-line $[0, \infty)$, so if $z \notin(-\infty, 0]$ and if the Laplace transform $\hat{f}(z)$ exists, then

$$
\hat{\mathrm{u}}(z)=(z \mathrm{I}+\mathrm{A})^{-1} \mathrm{~g}(z) .
$$

When $\hat{f}(z)$ is analytic and bounded for $\mathfrak{R} z>0$, the solution $\boldsymbol{u}(\mathbf{t})$ can be recovered via the Laplace inversion formula

$$
u(t)=\frac{1}{2 \pi i} \int_{\Gamma_{0}} e^{z t} \hat{u}(z) d z, \quad \text { for } t>0,
$$

where $\Gamma_{0}$ is the contour $\mathfrak{R} z=\omega$, for any $\omega>0$, with $\mathfrak{I} z$ increasing.

Section 2 summarizes some technical results and assumptions needed for our subsequent analysis. In Section 3 we describe the time discretization and quote a known error estimate (Theorem 3.1), after which we introduce 
the space discretization using SRBFs. The heart of the paper is Section 4 , where we prove two error bounds for the space discretization by adapting the analysis of Thomée 13 for a finite element approximation of the heat equation on a domain in $\mathbb{R}^{n}$. The first bound (Theorem 4.5) requires some spatial regularity of $\boldsymbol{u}_{0}$ and $f$, and is proved by estimating a contour integral. The second bound is proved by an energy argument, and assumes $f \equiv 0$ but allows nonsmooth initial data $\mathfrak{u}_{0} \in \mathrm{L}_{2}\left(\mathbb{S}^{n}\right)$. Both bounds include a factor that blows up as $t \rightarrow 0$. Finally, Section 5 describes the results of some numerical experiments.

\section{Preliminaries}

\section{$2.1 \quad$ Resolvent estimates}

We now view $A$ as an abstract, densely defined, self-adjoint and positivesemidefinite linear operator on a complex Hilbert space $\mathcal{H}$. Assume further that $(\mathrm{I}+\mathrm{A})^{-1}: \mathcal{H} \rightarrow \mathcal{H}$ is compact, so $A$ has a discrete spectrum, and order the eigenvalues $0 \leqslant \lambda_{1} \leqslant \lambda_{2} \leqslant \cdots$. Note that $\lambda_{j} \rightarrow \infty$ as $j \rightarrow \infty$ if $\mathcal{H}$ is infinite dimensional.

For any $\varphi>0$, the spectrum of $A$ is a subset of a closed sector in the complex plane $\mathbb{C}$,

$$
\Sigma_{\varphi}:=\{z \neq 0:|\arg z| \leqslant \varphi\} \cup\{0\}, \quad \text { with } 0<\varphi<\pi / 2 .
$$

In addition, there is a constant $C>0$ such that $A$ satisfies the resolvent estimate $\left\|(z \mathrm{I}-A)^{-1}\right\| \leqslant C|z|^{-1}$ for $z \in \overline{\mathbb{C} \backslash \Sigma_{\varphi}}$, or, equivalently,

$$
\left\|(z \mathrm{I}+\mathrm{A})^{-1}\right\| \leqslant C|z|^{-1}, \quad \text { for } z \in \Sigma_{\pi-\varphi},
$$

where $\|\cdot\|$ denotes the operator norm induced by the norm in $\mathcal{H}$.

\subsection{Sobolev spaces on the unit sphere}

Denote the inner product in $\mathcal{H}=\mathrm{L}_{2}\left(\mathbb{S}^{n}\right)$ by

$$
\langle v, w\rangle:=\int_{\mathbb{S}^{n}} v w \mathrm{~d} S
$$

where $\mathrm{d} S$ is the surface measure on the unit sphere, and denote the measure of the whole sphere by $\omega_{\mathrm{n}}$ (so, for example, $\omega_{2}=4 \pi$ ). Recall [6] 
that a spherical harmonic is the restriction to $\mathbb{S}^{n}$ of a homogeneous polynomial $Y(x)$ in $\mathbb{R}^{n+1}$ satisfying $\triangle Y(x)=0$, where $\triangle$ is the Laplacian operator in $\mathbb{R}^{n+1}$. The space of spherical harmonics of degree $\ell$, denoted by $\mathcal{H}_{\ell}$, has dimension $N(n, \ell):=\operatorname{dim} \mathcal{H}_{\ell}$, given by

$$
N(n, 0)=1 \text { and } N(n, \ell)=\frac{(2 \ell+n-1)(\ell+n-2) !}{\ell !(n-1) !} \text { for } \ell \geqslant 1 .
$$

In the usual way, we construct an orthonormal basis $\left\{Y_{\ell k}: 1 \leqslant k \leqslant N(n, \ell)\right\}$ for $\mathcal{H}_{\ell}$, so that $\left\langle\mathrm{Y}_{\ell k}, \mathrm{Y}_{\ell^{\prime} \mathrm{k}^{\prime}}\right\rangle=\delta_{\ell \ell^{\prime}} \delta_{\mathrm{kk}^{\prime}}$.

The Laplace-Beltrami operator $\triangle^{*}$ on $\mathbb{S}^{n}$ may be defined in terms of the Laplacian $\triangle$ on $\mathbb{R}^{n+1}$ by

$$
\triangle^{*} v=\left.\triangle \check{v}\right|_{\mathbb{S}^{n}} \quad \text { where } \quad \check{v}(x)=v(x /|x|)
$$

The spherical harmonics are eigenfunctions of $\triangle^{*}$, satisfying

$$
-\triangle^{*} Y_{\ell k}=\lambda_{\ell} Y_{\ell k} \quad \text { where } \quad \lambda_{\ell}=\ell(\ell+n-1),
$$

for $1 \leqslant k \leqslant N(n, \ell)$ and $\ell \in\{0,1,2, \ldots\}$. Every function $v \in \mathrm{L}_{2}\left(\mathbb{S}^{n}\right)$ can be expanded in a generalized Fourier series

$$
v=\sum_{\ell=0}^{\infty} \sum_{k=1}^{\mathrm{N}(\mathrm{n}, \ell)} \hat{v}_{\ell k} \mathrm{Y}_{\ell k} \quad \text { where } \quad \hat{v}_{\ell k}=\left\langle v, \mathrm{Y}_{\ell k}\right\rangle
$$

and for $\sigma \in \mathbb{R}$ we can characterize the Sobolev space on the unit sphere, $\mathrm{H}^{\sigma}=\mathrm{H}^{\sigma}\left(\mathbb{S}^{n}\right)$, in terms of the generalized Fourier coefficients: $v \in \mathrm{H}^{\sigma}$ if and only if the norm defined by

$$
\|v\|_{H^{\sigma}}^{2}:=\left\|\left(I-\Delta^{*}\right)^{\sigma / 2} v\right\|^{2}=\sum_{\ell=0}^{\infty}\left(1+\lambda_{\ell}\right)^{\sigma} \sum_{k=1}^{N(n, \ell)}\left|\hat{v}_{\ell k}\right|^{2}
$$

is finite. We also define the subspace of functions with mean zero,

$$
\mathrm{H}_{0}^{\sigma}=\mathrm{H}_{0}^{\sigma}\left(\mathbb{S}^{n}\right):=\left\{v \in \mathrm{H}^{\sigma}\left(\mathbb{S}^{n}\right): \int_{\mathbb{S}^{n}} v \mathrm{~d} S=0\right\} ;
$$

since $Y_{01}=1 / \sqrt{\omega_{n}}$ is constant, we see that $v \in H^{\sigma}$ belongs to $H_{0}^{\sigma}$ if and only if $\hat{v}_{01}=0$. 


\subsection{Positive definite kernels on the unit sphere}

A continuous function $\Phi: \mathbb{S}^{n} \times \mathbb{S}^{n} \rightarrow \mathbb{R}$ is called a positive definite kernel $[10$, 17] on $\mathbb{S}^{n}$ if it satisfies the following two conditions:

(i) $\Phi(x, y)=\Phi(y, x)$ for all $x, y \in \mathbb{S}^{n}$;

(ii) for any set of distinct scattered points $\left\{y_{1}, y_{2}, \ldots, y_{K}\right\} \subset \mathbb{S}^{n}$, the symmetric $\mathrm{K} \times \mathrm{K}$ matrix $\left[\Phi\left(\mathrm{y}_{i}, \mathrm{y}_{j}\right)\right]$ is positive semi-definite.

We call $\Phi$ strictly positive definite if the matrix is strictly positive definite.

We will work with a kernel $\Phi$ defined in terms of a univariate function $\phi$ : $[-1,1] \rightarrow \mathbb{R}$ by

$$
\Phi(x, y)=\phi(x \cdot y) \quad \text { for all } x, y \in \mathbb{S}^{n}
$$

where $x \cdot y$ denotes the Euclidean inner product of $x$ and $y$. Following Müller [6], let $P_{\ell}(t)$ denote the Legendre polynomial of degree $\ell$ for $\mathbb{R}^{n+1}$, and expand $\phi(t)$ in a Fourier-Legendre series

$$
\phi(t)=\frac{1}{\omega_{n}} \sum_{\ell=0}^{\infty} N(n, \ell) a_{\ell} P_{\ell}(t)
$$

Due to the addition formula for spherical harmonics [6, Page 10],

$$
\sum_{k=1}^{N(n, \ell)} Y_{\ell k}(x) Y_{\ell k}(y)=\frac{N(n, \ell)}{\omega_{n}} P_{\ell}(x \cdot y)
$$

the kernel $\Phi$ can be represented as

$$
\Phi(x, y)=\sum_{\ell=0}^{\infty} \sum_{k=1}^{N(n, \ell)} a_{\ell} Y_{\ell k}(x) Y_{\ell k}(y)
$$

and since $P_{\ell}(1)=1$ we find that

$$
\|\Phi(x, \cdot)\|_{H^{\tau}}^{2}=\frac{1}{\omega_{n}} \sum_{\ell=0}^{\infty}\left(1+\lambda_{\ell}\right)^{\tau} a_{\ell}^{2} N(n, \ell), \quad \text { for all } x \in \mathbb{S}^{n} .
$$

Chen et al. [2] proved that the kernel $\Phi$ is strictly positive definite if and only if $a_{\ell} \geqslant 0$ for all $\ell \geqslant 0$ and $a_{\ell}>0$ for infinitely many even values of $\ell$ 
and infinitely many odd values of $\ell$; see also Schoenberg [10] and $\mathrm{Xu}$ and Cheney [17]. Here, we assume there is a $\tau>n / 2$ and positive constants $\mathrm{c}$ and $\mathrm{C}$ such that

$$
c\left(1+\lambda_{\ell}\right)^{-\tau} \leqslant a_{\ell} \leqslant C\left(1+\lambda_{\ell}\right)^{-\tau}, \quad \text { for all } \ell \geqslant 0
$$

Hence, $\Phi$ is strictly positive definite and, since $\mathrm{N}(\mathrm{n}, \ell)=\mathrm{O}\left(\ell^{n-1}\right)$ as $\ell \rightarrow \infty$, the sum (2.7) is finite so, for each fixed $x \in \mathbb{S}^{n}$, the function $y \mapsto \Phi(x, y)$ belongs to $\mathrm{H}^{\tau}\left(\mathbb{S}^{n}\right)$. Moreover, this function is continuous by the Sobolev imbedding theorem.

\section{The discrete problem}

Choose an angle $\beta \in(\pi / 2, \pi-\varphi)$ and let $\Gamma$ be any curve in the interior of the sector $\Sigma_{\beta}$ which is homotopic to the line $\Gamma_{0}$ appearing in the Laplace inversion formula (1.5). Deforming the contour of integration in (1.5), we may then write

$$
u(t)=\frac{1}{2 \pi i} \int_{\Gamma} e^{z t} \hat{u}(z) d z
$$

assuming that $\hat{f}(z)$ is analytic on and to the right of $\Gamma$.

By taking $f \equiv 0$ in (1.1), so that $g(z)=u_{0}$ in (1.3), we see that the solution operator for the homogeneous problem has the integral representation

$$
\mathcal{E}(\mathrm{t}) \mathrm{u}_{0}=\frac{1}{2 \pi i} \int_{\Gamma} e^{z \mathrm{t}} \hat{\mathcal{E}}(z) u_{0} \mathrm{~d} z, \quad \text { where } \hat{\mathcal{E}}(z)=(z \mathrm{I}+\mathrm{A})^{-1}
$$

For the inhomogeneous case, the inverse Laplace transform of $\hat{\mathcal{E}}(z) \hat{f}(z)$ is the convolution of $\mathcal{E}(t)$ and $f(t)$, giving the Duhamel formula

$$
u(t)=\mathcal{E}(t) u_{0}+\int_{0}^{t} \mathcal{E}(t-s) f(s) d s .
$$

A standard energy argument shows that $\left\|\mathcal{E}(t) u_{0}\right\| \leqslant\left\|\mathfrak{u}_{0}\right\|$ for all $t \geqslant 0$, so the continuous problem (1.1) is stable in the sense that

$$
\|u(t)\| \leqslant\left\|u_{0}\right\|+\int_{0}^{t}\|f(s)\| d s, \quad \text { for } t \geqslant 0 .
$$


For our numerical methods we choose $\Gamma$ to be the curve with parametric representation

$$
z(\xi):=\omega+\lambda(1-\sin (\delta-i \xi)), \quad \text { for } \xi \in \mathbb{R},
$$

where the constants $\omega, \lambda$ and $\delta$ satisfy

$$
\omega>0, \quad \lambda>0 \text { and } 0<\delta<\beta-\pi / 2 \text {. }
$$

Writing $z=x+i y$, we find that $\Gamma$ is the left branch of the hyperbola

$$
\left(\frac{x-\omega-\lambda}{\lambda \sin \delta}\right)^{2}-\left(\frac{y}{\lambda \cos \delta}\right)^{2}=1,
$$

which cuts the real axis at the point $z=\omega+\lambda(1-\sin \delta)$ and has asymptotes $y= \pm(x-\omega-\lambda) \cot \delta$. Thus, the conditions (3.5) ensure that $\Gamma$ lies in the sector $\Sigma_{\beta}^{\omega}:=\omega+\Sigma_{\beta} \subset \Sigma_{\beta}$, and crosses into the left half-plane.

We use (3.4) in (3.1) to represent $u(t)$ as an integral with respect to $\xi$,

$$
u(t)=\frac{1}{2 \pi i} \int_{-\infty}^{\infty} e^{z(\xi) t} w(z(\xi)) z^{\prime}(\xi) d \xi
$$

Since $\left|e^{z(\xi) t}\right|=e^{\Re z(\xi) t}=e^{\omega t} e^{\lambda t(1-\sin \delta \cosh \xi)}$, the integrand exhibits a double exponential decay as $|\xi| \rightarrow \infty$, for any fixed $t>0$.

\subsection{Time discretization}

We choose a quadrature step $k$, put

$$
\xi_{j}:=j k, \quad z_{j}:=z\left(\xi_{j}\right), \quad z_{j}^{\prime}:=z^{\prime}\left(\xi_{j}\right),
$$

and apply an equal weight rule to the integral (3.7) to obtain an approximate solution

$$
\mathrm{u}_{\mathrm{N}}(\mathrm{t}):=\frac{\mathrm{k}}{2 \pi i} \sum_{j=-N}^{N} e^{z_{j} \mathrm{t}} \hat{\mathrm{u}}\left(z_{j}\right) z_{j}^{\prime} .
$$

In view of (3.1), to compute $\mathrm{U}_{\mathrm{N}}(\mathrm{t})$ we must solve the $2 \mathrm{~N}+1$ equations

$$
\left(z_{j} I+A\right) \hat{u}\left(z_{j}\right)=g\left(z_{j}\right), \quad \text { for }|j| \leqslant N .
$$

These equations are independent and hence may be solved in parallel. Notice that the $\hat{u}\left(z_{\mathfrak{j}}\right)$ determine the approximate solution (3.8) for all $t>0$ and that 
the numerical solution (3.8) depends on the choice of the curve $\Gamma$, even though the representation (3.1) does not. However, we will see that a given $\Gamma$ and $\mathrm{k}$ yield an accurate approximation $\mathrm{U}_{\mathrm{N}}(\mathrm{t}) \approx \mathrm{u}(\mathrm{t})$ only for $\mathrm{t}$ at a particular time scale.

The parametric representation (3.4) of $\Gamma$ extends to a conformal mapping

$$
z=\Psi(\zeta)=\omega+\lambda(1-\sin (\delta-i \zeta))
$$

which, for $r>0$, transforms the strip $Y_{r}:=\{\zeta:|\Im \zeta| \leqslant r\}$ onto the set $S_{r}:=$ $\left\{\Psi(\zeta): \zeta \in Y_{\mathrm{r}}\right\} \supset \Gamma$. In fact, $\Psi$ maps the line $\mathfrak{I} \zeta=\eta$ to the left branch of a hyperbola given by (3.6) with $\delta$ replaced by $\delta+\eta$. Thus, $S_{\mathrm{r}}$ is bounded by the left branches of the hyperbolas corresponding to $\mathfrak{I} \zeta=r$ and $\mathfrak{I} \zeta=-r$. To ensure that $S_{\mathrm{r}} \subset \Sigma_{\beta}^{\omega}$ and that $\mathfrak{R} z \rightarrow-\infty$ if $|z| \rightarrow \infty$ with $z \in S_{\mathrm{r}}$, we require $0<\delta-r<\delta+r<\beta-\pi / 2$, or equivalently that

$$
0<r<\min (\delta, \beta-\pi / 2-\delta)
$$

We introduce the notation

$$
\|g\|_{X, Z}:=\sup _{z \in Z}\|g(z)\|_{X}, \quad \text { for } X \subseteq \mathcal{H} \text { and } Z \subseteq \mathbb{C}
$$

abbreviated by $\|\mathrm{g}\|_{\mathrm{z}}$ if $\mathrm{X}=\mathcal{H}$, and put $\lg (\mathrm{s})=\max (1, \log (1 / \mathrm{s}))$.

Theorem 3.1. Let $u$ be the solution of (1.1), with $\hat{f}$ bounded and analytic in $\Sigma_{\beta}^{\omega}$, and fix a time scale $\mathrm{T}>0$. Let $0<\theta<1$ and define $\mathrm{b}>0$ by $\cosh \mathrm{b}=4 /(\theta \sin \delta)$, let $\mathrm{r}$ satisfy (3.11) so that $\Gamma \subset \mathrm{S}_{\mathrm{r}} \subset \Sigma_{\beta}^{\omega}$, and put $\lambda=\pi \mathrm{r} \theta \mathrm{N} /(\mathrm{bT})$. Then the approximate solution $\mathrm{U}_{\mathrm{N}}(\mathrm{t})$ defined by (3.8) with $\mathrm{k}=\mathrm{b} / \mathrm{N} \leqslant 2 \pi \mathrm{r} \log 2$ satisfies

$\left\|\mathrm{U}_{\mathrm{N}}(\mathrm{t})-\mathrm{u}(\mathrm{t})\right\| \leqslant C e^{\omega t} \lg \left(\rho_{\mathrm{r}} \mathrm{N}\right) \mathrm{e}^{-\mu \mathrm{N}}\left(\left\|\mathrm{u}_{0}\right\|+\|\hat{\mathrm{f}}\|_{\Sigma_{\beta}^{\omega}}\right), \quad$ for $\mathrm{T} / 2 \leqslant \mathrm{t} \leqslant 2 \mathrm{~T}$,

where $\mu=2 \pi r(1-\theta) / b, \rho_{r}=\pi r \theta \sin (\delta-r) /(2 b)$ and $C=C_{\delta, r, \beta}$.

Proof. See McLean and Thomée [5, Theorem 3.1].

\subsection{Galerkin approximation by SRBFs}

Given a suitable set of points $X=\left\{x_{1}, x_{2}, \ldots, x_{K}\right\} \subseteq \mathbb{S}^{n}$ and a strictly positive definite kernel $\Phi(x, y)$, we define the spherical radial basis functions $\Phi_{p}(x):=$ 
$\Phi\left(x_{p}, x\right)$ for $1 \leqslant p \leqslant K$. Recall that our assumption (2.8) ensures $\Phi_{p} \in H^{\tau}$ with $\tau>n / 2 \geqslant 1$; thus

$$
\mathrm{S}_{\mathrm{h}}:=\operatorname{span}\left\{\Phi_{\mathrm{p}}: 1 \leqslant \mathrm{p} \leqslant \mathrm{K}\right\} \subseteq \mathrm{H}^{1} .
$$

The uniformity of the set $X$ is measured by its mesh norm $h_{X}$ and its separation radius $q_{x}$, defined by

$$
h=h_{X}:=\sup _{y \in \mathbb{S}^{n}} \min _{x \in X} \cos ^{-1}(y \cdot x) \text { and } q=q_{x}:=\frac{1}{2} \min _{\substack{x \neq y \\ x, y \in X}} \cos ^{-1}(y \cdot x) .
$$

In words, $h_{X}$ is the maximum geodesic distance from a point on $\mathbb{S}^{n}$ to the nearest point of $X$. For our convergence analysis, we require that the family of point sets $\{X\}$ has a bounded mesh ratio:

$$
h_{x} \leqslant \mathrm{Cq}_{\mathrm{x}}
$$

Associated with the second-order, partial differential differential operator $A$ is a bounded sesquilinear form $\mathrm{a}: \mathrm{H}^{1} \times \mathrm{H}^{1} \rightarrow \mathbb{C}$ defined by

$$
a(u, v)=\langle A u, v\rangle \quad \text { for } u, v \in H^{1} \text {. }
$$

For example, if $A=-\Delta^{*}$ then $a(u, v)=\langle\operatorname{grad} u, \operatorname{grad} v\rangle$ where $\operatorname{grad}$ is the surface gradient. The mild solution $\mathfrak{u}:[0, \infty) \rightarrow \mathrm{L}_{2}\left(\mathbb{S}^{n}\right)$ of (1.1) satisfies

$$
\left\langle\partial_{t} u, v\right\rangle+a(u, v)=\langle f(t), v\rangle \quad \text { for } t>0 \text { and all } v \in H^{1}
$$

with $\mathfrak{u}(0)=\mathfrak{u}_{0}$, and we define a semidiscrete solution $\mathfrak{u}_{\mathrm{h}}:[0, \infty) \rightarrow \mathrm{S}_{\mathrm{h}}$ of (1.1) by

$$
\left\langle\partial_{t} u_{h}, \chi\right\rangle+a\left(u_{h}, \chi\right)=\langle f(t), \chi\rangle \quad \text { for all } \chi \in S_{h}
$$

with $\mathfrak{u}_{\mathrm{h}}(0)=\mathfrak{u}_{0 \mathrm{~h}} \approx \mathfrak{u}_{0}$ for a suitable $\mathfrak{u}_{0 \mathrm{~h}} \in \mathrm{S}_{\mathrm{h}}$.

The Laplace transform of $u$ at $z_{j}$ is the weak solution $\hat{u}\left(z_{j}\right) \in \mathrm{H}^{1}$ of (3.9), that is,

$$
z_{j}\left\langle\hat{u}\left(z_{j}\right), v\right\rangle+a\left(\hat{u}\left(z_{j}\right), v\right)=\left\langle g\left(z_{j}\right), v\right\rangle \text { for all } v \in H^{1},
$$

and the Laplace transform of the semidiscrete solution, $\hat{\mathfrak{u}}_{\mathrm{h}}\left(z_{j}\right) \in \mathrm{S}_{\mathrm{h}}$, satisfies

$$
z_{j}\left\langle\hat{u}_{h}\left(z_{j}\right), \chi\right\rangle+a\left(\hat{u}_{h}\left(z_{j}\right), \chi\right)=\left\langle g_{h}\left(z_{j}\right), \chi\right\rangle \text { for all } \chi \in S_{h},
$$


where $g_{h}(z)=u_{0 h}+P_{h} \hat{f}(z) \in S_{h}$ and $P_{h}$ denotes the orthogonal projector from $L_{2}\left(\mathbb{S}^{n}\right)$ onto $S_{h}$. Thus, we can view $\hat{u}_{h}\left(z_{j}\right)$ as a Galerkin approximation to $\hat{u}\left(z_{j}\right)$. Concretely, to compute $\hat{u}_{h}(z)=\sum_{p=1}^{K} \hat{U}_{p}(z) \Phi_{p}$ we form the $K \times K$ matrices $B$ and $S$, with entries

$$
\mathrm{B}_{\mathrm{pq}}=\left\langle\Phi_{\mathrm{p}}, \Phi_{\mathrm{q}}\right\rangle \text { and } \mathrm{S}_{\mathrm{pq}}=\mathrm{a}\left(\Phi_{\mathrm{p}}, \Phi_{\mathrm{q}}\right)
$$

form the load vector $\mathbf{G}(z) \in \mathbb{C}^{\mathrm{K}}$ with components $\mathrm{G}_{\mathrm{p}}(z)=\left\langle\mathrm{g}_{\mathrm{h}}(z), \Phi_{\mathrm{p}}\right\rangle$, and then solve the $\mathrm{K} \times \mathrm{K}$ complex linear system

$$
\left(z_{j} B+S\right) \hat{\mathbf{U}}\left(z_{j}\right)=\mathbf{G}\left(z_{j}\right),
$$

to obtain the solution vector $\hat{\mathbf{U}}(z) \in \mathbb{C}^{\mathrm{K}}$ with components $\hat{\mathbf{U}}_{\mathrm{p}}(z)$. In contrast to finite element mass and stiffness matrices, $B$ and $S$ are not sparse because the SRBFs have large supports.

\subsection{Fully-discrete solution}

Combining the time and space discretizations, we arrive at a fully-discrete solution

$$
\mathrm{u}_{\mathrm{N}, \mathrm{h}}(\mathrm{t})=\frac{\mathrm{k}}{2 \pi i} \sum_{j=-\mathrm{N}}^{\mathrm{N}} e^{z_{j} \mathrm{t}} \hat{\mathrm{u}}_{\mathrm{h}}\left(z_{\mathrm{j}}\right) z_{j}^{\prime},
$$

whose evaluation requires that we solve the linear system (3.16) at each of the $2 \mathrm{~N}+1$ quadrature points $z_{j}$. (In practice, we also use quadratures for the integrations over $\mathbb{S}^{n}$ that are needed to compute $B_{p q}, S_{p q}$ and $G_{p}(z)$, but for our analysis we assume that these quantities are computed exactly.) The elliptic differential operator $A$ induces a discrete operator $A_{h}: S_{h} \rightarrow S_{h}$, defined by

$$
\left\langle A_{h} \psi, \chi\right\rangle=a(\psi, \chi), \quad \text { for } \psi, \chi \in S_{h},
$$

and the Galerkin equations (3.14) are equivalent to

$$
\left(z_{j} I+A_{h}\right) \hat{u}_{h}\left(z_{j}\right)=g_{h}\left(z_{j}\right) .
$$

If we choose $u_{0 h}=P_{h} u_{0}$ then $g_{h}\left(z_{j}\right)=P_{h} g\left(z_{j}\right)$ and by taking $\mathcal{H}=S_{h}$ equipped with the $L_{2}$-norm, we can apply Theorem 3.1 to $A_{h}$ and deduce that

$\left\|\mathrm{u}_{\mathrm{N}, \mathrm{h}}(\mathrm{t})-\mathrm{u}_{\mathrm{h}}(\mathrm{t})\right\| \leqslant C e^{\omega t} \lg \left(\rho_{\mathrm{r}} \mathrm{N}\right) e^{-\mu \mathrm{N}}\left(\left\|\mathrm{u}_{0}\right\|+\|\hat{\mathrm{f}}\|_{\Sigma_{\beta}^{\omega}}\right), \quad$ for $\mathrm{T} / 2 \leqslant \mathrm{t} \leqslant 2 \mathrm{~T}$. 
Since the triangle inequality gives

$$
\left\|\mathrm{U}_{\mathrm{N}, \mathrm{h}}(\mathrm{t})-\mathrm{u}(\mathrm{t})\right\| \leqslant\left\|\mathrm{U}_{\mathrm{N}, \mathrm{h}}(\mathrm{t})-\mathrm{u}_{\mathrm{h}}(\mathrm{t})\right\|+\left\|\mathrm{u}_{\mathrm{h}}(\mathrm{t})-\mathrm{u}(\mathrm{t})\right\|
$$

to estimate the error in $\mathbf{U}_{\mathrm{N}, \mathrm{h}}$ it now suffices to estimate the error in the semidiscrete approximation $\mathfrak{u}_{\mathrm{h}}(\mathrm{t})$.

\section{Error analysis of the spatial discretization}

We assume now that $A=-\triangle^{*}$. Since $\lambda_{0}=0$ but $\lambda_{\ell} \geqslant \lambda_{1}=\mathrm{n}$ for all $\ell \geqslant 1$, we see that $1+\lambda_{\ell} \leqslant\left(1+n^{-1}\right) \lambda_{\ell}$ for all $\ell \geqslant 1$. Hence, the sesquilinear form $a$ is coercive on $\mathrm{H}_{0}^{1}$, that is,

$$
a(v, v) \geqslant \frac{\|v\|_{\mathrm{H}^{1}}^{2}}{1+\mathrm{n}^{-1}} \quad \text { if } v \in \mathrm{H}^{1} \text { and } \hat{v}_{10}=\int_{\mathbb{S}^{n}} v \mathrm{~d} S=0
$$

Our analysis follows Thomée [13, Chapter 3], with $\triangle^{*}$ in place of the Laplacian (with homogeneous Dirichlet boundary conditions). Some technical modifications are needed, however, because $\triangle^{*}$ has a zero eigenvalue.

\subsection{Approximation by SRBFs}

We will use the following estimate for the best approximation by SRBFs.

Theorem 4.1. Assume that the Fourier-Legendre coefficients in the expansion (2.5) satisfy (2.8) with $\tau>\mathrm{n} / 2$, so that $\mathrm{S}_{\mathrm{h}} \subseteq \mathrm{H}^{\tau}\left(\mathbb{S}^{\mathrm{n}}\right)$. For any real $\mathrm{q}$ and $\boldsymbol{v}$ satisfying $\mathrm{q} \leqslant \boldsymbol{v} \leqslant 2 \tau$ and $\mathrm{q} \leqslant \tau$, if $\boldsymbol{v} \in \mathrm{H}^{v}$ then there exists $\chi \in \mathrm{S}_{\mathrm{h}}$ such that

$$
\|\chi-v\|_{H^{q}} \leqslant C h_{X}^{v-q}\|v\|_{H^{v}} .
$$

Proof. See Tran et al. [15, Theorem 3.2] or [14, Theorem 3.7 and Remark 5.1], and note our assumption (3.12).

In the special case $q=0$, the estimate must hold for $\chi=P_{h} v$, giving the following result.

Corollary 4.2. The $\mathrm{L}_{2}$-projection of $\mathrm{v}$ onto $\mathrm{S}_{\mathrm{h}}$ has the approximation property

$$
\left\|v-\mathrm{P}_{\mathrm{h}} v\right\| \leqslant \mathrm{Ch}_{\mathrm{X}}^{v}\|v\|_{\mathrm{H}^{v}} \quad \text { for } 0 \leqslant v \leqslant 2 \tau
$$


For our error analysis, we also use the Ritz projector $R_{h}: H^{1}\left(\mathbb{S}^{n}\right) \rightarrow S_{h}$ determined by the sesquilinear form

$$
\mathrm{a}_{1}(u, v)=\mathrm{a}(\mathrm{u}, v)+\langle u, v\rangle \quad \text { for } u, v \in \mathrm{H}^{1} .
$$

We see from (4.1) that $\mathrm{a}_{1}$ is coercive on $\mathrm{H}^{1}$; in fact, $\mathrm{a}_{1}(v, v)=\|v\|_{\mathrm{H}^{1}}^{2}$. Thus, $R_{h} \nu \in S_{h}$ is well-defined by

$$
a_{1}\left(R_{h} v, \chi\right)=a_{1}(v, \chi) \text { for all } \chi \in S_{h},
$$

and the following error estimates hold using standard arguments.

Theorem 4.3. If $v \in \mathrm{H}^{v}$ and $1 \leqslant v \leqslant 2 \tau$, then

$$
\left\|v-\mathrm{R}_{\mathrm{h}} v\right\|_{\mathrm{H}^{1}}=\inf _{\chi \in \mathrm{S}_{\mathrm{h}}}\|v-\chi\|_{\mathrm{H}^{1}} \leqslant \mathrm{Ch}_{\mathrm{X}}^{\nu-1}\|v\|_{\mathrm{H}^{v}}
$$

and

$$
\left\|v-\mathrm{R}_{\mathrm{h}} v\right\| \leqslant \mathrm{Ch}_{\mathrm{X}}^{v}\|v\|_{\mathrm{H}^{v}}
$$

Proof. The definition (4.2) immediately implies the orthogonality property

$$
a_{1}\left(v-R_{h} v, \chi\right)=0 \quad \text { for all } \chi \in S_{h},
$$

so, because $a_{1}(v, v)=\|v\|_{\mathrm{H}^{1}}^{2}$,

$$
\begin{aligned}
\left\|v-\mathrm{R}_{\mathrm{h}} v\right\|_{\mathrm{H}^{1}}^{2} & =\mathrm{a}_{1}\left(v-\mathrm{R}_{\mathrm{h}} v, v-\mathrm{R}_{\mathrm{h}} v\right)=\mathrm{a}_{1}\left(v-\mathrm{R}_{\mathrm{h}} v, v-\chi\right) \\
& \leqslant\left\|v-\mathrm{R}_{\mathrm{h}} v\right\|_{\mathrm{H}^{1}}\|v-\chi\|_{\mathrm{H}^{1}},
\end{aligned}
$$

and thus $\left\|v-R_{h} v\right\|_{H^{1}} \leqslant\|v-\chi\|_{H^{1}}$ for all $\chi \in S_{h}$. The first claim now follows by Theorem 4.1 .

A duality argument [8] yields the second claim. Given $v$ there is a unique $u \in H^{1}$ satisfying $(I+A) u=v-R_{h} v$, or equivalently (since $A$ is self-adjoint)

$$
\mathrm{a}_{1}(w, u)=\left\langle w, v-\mathrm{R}_{\mathrm{h}} v\right\rangle \quad \text { for all } w \in \mathrm{H}^{1},
$$

Taking $w=v-R_{h} v$ and applying (4.3), we have for every $\chi \in S_{h}$,

$$
\begin{aligned}
\left\langle v-\mathrm{R}_{\mathrm{h}} v, v-\mathrm{R}_{\mathrm{h}} v\right\rangle & =\mathrm{a}_{1}\left(v-\mathrm{R}_{\mathrm{h}} v, \mathrm{u}\right)=\mathrm{a}_{1}\left(v-\mathrm{R}_{\mathrm{h}} v, \mathrm{u}-\chi\right) \\
& \leqslant\left\|v-\mathrm{R}_{\mathrm{h}} v\right\|_{\mathrm{H}^{1}}\|\mathrm{u}-\chi\|_{\mathrm{H}^{1}} \leqslant \mathrm{Ch}^{v-1}\|v\|_{\mathrm{H}^{v}}\|\mathrm{u}-\chi\|_{\mathrm{H}^{1}} .
\end{aligned}
$$

By Theorem 4.1 with $\mathrm{q}=1$ and $v=2 \leqslant 2 \tau$, there is a $\chi \in S_{\mathrm{h}}$ such that $\|\mathrm{u}-\chi\|_{\mathrm{H}^{1}} \leqslant \mathrm{Ch}\|\mathrm{u}\|_{\mathrm{H}^{2}}$, so

$$
\left\|v-R_{h} v\right\|^{2} \leqslant C h^{v}\|v\|_{H^{v}}\|u\|_{H^{2}}
$$

and the result follows because $\|u\|_{H^{2}}=\|(I+A) u\|=\left\|v-R_{h} v\right\|$. 


\subsection{Contour integral estimate}

We see from (1.3) and (3.19) that, assuming $\mathbf{u}_{0 \mathrm{~h}}=\mathrm{P}_{\mathrm{h}} \mathbf{u}_{0}$,

$$
\hat{u}(z)=(z I+A)^{-1} g(z) \text { and } \hat{\mathfrak{u}}_{h}(z)=\left(z I+A_{h}\right)^{-1} P_{h} g(z),
$$

SO

$$
\hat{u}_{h}(z)-\hat{u}(z)=G_{h}(z) g(z) \quad \text { where } \quad G_{h}(z):=\left(z I+A_{h}\right)^{-1} P_{h}-(z I+A)^{-1} .
$$

Deforming the integration contour in the Laplace inversion formula to $\Gamma=$ $\partial \Sigma_{\beta}^{\omega}$, we can represent the error in the semidiscrete solution as follows:

$$
u_{h}(t)-u(t)=\frac{1}{2 \pi i} \int_{\Gamma} e^{z t} G_{h}(z) g(z) d z .
$$

The next lemma allows us to estimate this integral.

Lemma 4.4. If $0 \leqslant v \leqslant 2 \tau$, then

$$
\left\|\mathrm{G}_{\mathrm{h}}(z) v\right\| \leqslant \mathrm{Ch}_{\mathrm{X}}^{v}\|v\|_{\mathrm{H}^{v-2}}, \quad \text { for } z \in \Sigma_{\beta}^{\omega} \text { and } v \in \mathrm{H}^{v-2} .
$$

Proof. Recall that $\hat{\mathcal{E}}(z):=(z \mathrm{I}+A)^{-1}$, and let $\hat{\mathcal{E}}_{\mathrm{h}}(z):=\left(z \mathrm{I}+\mathrm{A}_{\mathrm{h}}\right)^{-1}$. We split $\mathrm{G}_{\mathrm{h}}(z)$ into two terms,

$$
\mathrm{G}_{h}(z)=\left(\mathrm{P}_{h}-\mathrm{I}\right) \hat{\mathcal{E}}(z)+\left[\hat{\mathcal{E}}_{h}(z) \mathrm{P}_{h}-\mathrm{P}_{\mathrm{h}} \hat{\mathcal{E}}(z)\right] .
$$

Since $A \hat{\varepsilon}(z)=(z I+A-z I)(z I+A)^{-1}=I-z(z I+A)^{-1}$, the resolvent estimate (2.1) shows that

$$
\|A \hat{\varepsilon}(z) v\| \leqslant C\|v\| \quad \text { for } z \in \Sigma_{\beta}^{\omega} .
$$

Moreover, since $(I+A) \hat{\mathcal{E}}(z)=I+(1-z) \hat{\mathcal{E}}(z)$ and since $(I+A)^{1 / 2}$ commutes with $(\mathrm{I}+\mathrm{A}) \hat{\mathcal{E}}(z)$, we have $\|(\mathrm{I}+\mathrm{A}) \hat{\mathcal{E}}(z) v\|_{\mathrm{H}^{\mathrm{q}}} \leqslant \mathrm{C}\left|1-z\left\|\left.z\right|^{-1}\right\| v \|_{\mathrm{H}^{\mathrm{q}}}\right.$ for any $\mathrm{q} \in \mathbb{R}$, and thus by Corollary 4.2 ,

$\left\|\left(\mathrm{P}_{h}-\mathrm{I}\right) \hat{\mathcal{E}}(z) v\right\| \leqslant \mathrm{Ch}^{v}\|\hat{\mathcal{E}}(z) v\|_{\mathrm{H}^{v}}=\mathrm{Ch}^{v}\|(\mathrm{I}+\mathrm{A}) \hat{\mathcal{E}}(z) v\|_{\mathrm{H}^{v-2}} \leqslant \mathrm{Ch}^{v}\|v\|_{\mathrm{H}^{v-2}}$, noting that $|1-z||z|^{-1} \leqslant \mathrm{C}_{\omega, \beta}$ for $z \in \Sigma_{\beta}^{\omega}$.

To estimate the second term in (4.5), we write

$$
\begin{aligned}
\hat{\mathcal{E}}_{h}(z) P_{h}-P_{h} \hat{\mathcal{E}}(z) & =\hat{\mathcal{E}}_{h}(z) P_{h}(z I+A) \hat{\mathcal{E}}(z)-\hat{\mathcal{E}}_{h}(z)\left(z I+A_{h}\right) P_{h} \hat{\mathcal{E}}(z) \\
& =\hat{\mathcal{E}}_{h}(z)\left[P_{h} A-A_{h} P_{h}\right] \hat{\mathcal{E}}(z)
\end{aligned}
$$


For all $\mathfrak{u}, \mathfrak{w} \in \mathrm{H}^{1}$,

$$
\begin{aligned}
\left\langle P_{h}(I+A) u, w\right\rangle & =\left\langle(I+A) u, P_{h} w\right\rangle=a_{1}\left(u, P_{h} w\right)=a_{1}\left(R_{h} u, P_{h} w\right) \\
& =\left\langle\left(I+A_{h}\right) R_{h} u, P_{h} w\right\rangle=\left\langle\left(I+A_{h}\right) R_{h} u, w\right\rangle,
\end{aligned}
$$

so $P_{h}(I+A)=\left(I+A_{h}\right) R_{h}$ and thus

$$
\hat{\mathcal{E}}_{h}(z) \mathrm{P}_{\mathrm{h}}-\mathrm{P}_{\mathrm{h}} \hat{\mathcal{E}}(z)=\hat{\mathcal{E}}_{\mathrm{h}}(z)\left(\mathrm{I}+\mathrm{A}_{\mathrm{h}}\right) \mathrm{P}_{\mathrm{h}}\left(\mathrm{R}_{\mathrm{h}}-\mathrm{I}\right) \hat{\mathcal{E}}(z) .
$$

Since $\hat{\mathcal{E}}_{h}(z)\left(I+A_{h}\right)=I+(1-z) \hat{\mathcal{E}}_{h}(z)$ the resolvent estimate (2.1) and Theorem 4.3 imply that

$$
\begin{aligned}
\left\|\left[\hat{\mathcal{E}}_{h}(z) \mathrm{P}_{h}-\mathrm{P}_{h} \hat{\mathcal{E}}(z)\right] v\right\| \leqslant\left(1+\mathrm{C}|1-z \| z|^{-1}\right)\left\|\left(\mathrm{R}_{\mathrm{h}}-\mathrm{I}\right) \hat{\mathcal{E}}(z) v\right\| \\
\leqslant \mathrm{Ch}^{v}\|\hat{\mathcal{E}}(z) v\|_{\mathrm{H}^{v}}=\mathrm{Ch}^{v}\|(\mathrm{I}+\mathrm{A}) \hat{\mathcal{E}}(z) v\|_{\mathrm{H}^{v-2}} \leqslant \mathrm{Ch}^{v}\|v\|_{\mathrm{H}^{v-2}},
\end{aligned}
$$

noting again that $|1-z||z|^{-1} \leqslant \mathrm{C}_{\omega, \beta}$ for $z \in \Sigma_{\beta}^{\omega}$.

Theorem 4.5. Let $\mathfrak{u}$ be the solution of (1.1) and let $\mathfrak{u}_{\mathfrak{h}}$ be the semidiscrete approximation given by (3.13). If $0 \leqslant v \leqslant 2 \tau$, then

$$
\left\|\mathfrak{u}_{\mathfrak{h}}(\mathfrak{t})-\mathfrak{u}(\mathfrak{t})\right\| \leqslant \operatorname{Ch}_{X}^{v} \mathfrak{t}^{-1} e^{\omega t}\left(\left\|\mathfrak{u}_{0}\right\|_{H^{v-2}}+\|\hat{\mathfrak{f}}\|_{H^{v-2}, \partial \Sigma_{\beta}^{\omega}}\right), \quad \text { for } \mathbf{t}>0 .
$$

Proof. Let $\Gamma_{ \pm}$be the half-line $z=\omega+s e^{ \pm i \beta}$ for $0<s<\infty$, so that $\Gamma=\Gamma_{+}-\Gamma_{-}$. Since $\mathfrak{R z}=\omega-\mathrm{cs}$ where $c=-\cos \beta>0$, by applying Lemma 4.4 we have

$$
\begin{aligned}
\left\|\int_{\Gamma_{ \pm}} e^{z \mathfrak{t}} G_{h}(z) g(z) d z\right\| & \leqslant \int_{0}^{\infty} e^{(\omega-c s) t}\left\|G_{h}(z) g(z)\right\| d s \\
& \leqslant C e^{\omega t} h^{v}\|g\|_{H^{v-2}, \Gamma} \int_{0}^{\infty} e^{-c s t} d s
\end{aligned}
$$

and the error bound follows at once from the integral representation (4.4).

Combining Theorems 3.1 and 4.5, we conclude that provided $\mathfrak{u}_{0}$ and $f$ have the appropriate spatial regularity,

$$
\left\|\mathrm{U}_{\mathrm{N}, \mathrm{h}}(\mathrm{t})-\mathrm{u}(\mathrm{t})\right\|=\mathrm{O}\left(\lg \left(\rho_{\mathrm{r}} \mathrm{N}\right) \mathrm{e}^{-\mu \mathrm{N}}+\mathrm{h}_{\mathrm{X}}^{2 \tau}\right) \quad \text { for } \mathrm{T} / 2 \leqslant \mathrm{t} \leqslant 2 \mathrm{~T},
$$

where the constant includes a factor $\left(1+\mathrm{T}^{-1}\right) \mathrm{e}^{2 \omega \mathrm{T}}$. Moreover, in the next section (Theorem 4.8, Part 2) we will see that when $f \equiv 0$ the error bound (4.6) remains valid even if the initial data is not regular. 


\subsection{Nonsmooth initial data}

Consider the case $f \equiv 0$, that is,

$$
\partial_{t} u-\Delta^{*} u=0 \quad \text { on } \mathbb{S}^{n} \text { for } t>0, \quad \text { with } u=u_{0} \text { when } t=0,
$$

and the corresponding semidiscrete problem in which $u_{h}:[0, \infty) \rightarrow S_{h}$ satisfies

$$
\partial_{t} u_{h}-\triangle_{h}^{*} u_{h}=0 \quad \text { on } \mathbb{S}^{n} \text { for } t>0, \quad \text { with } u=u_{0 h} \text { when } t=0,
$$

where $\triangle_{\mathrm{h}}^{*}: \mathrm{S}_{\mathrm{h}} \rightarrow \mathrm{S}_{\mathrm{h}}$ is defined by

$$
\left\langle-\triangle_{h}^{*} \psi, \chi\right\rangle=a(\psi, \chi)=\langle\operatorname{grad} \psi, \operatorname{grad} \chi\rangle \text { for all } \psi, \chi \in S_{h} ;
$$

compare with (3.18). In contrast to the forgoing analysis, we now permit the initial data $\mathfrak{u}_{0}$ to be an arbitrary function in $\mathrm{L}_{2}\left(\mathbb{S}^{n}\right)$.

By separating variables, we obtain an expansion in spherical harmonics,

$$
u(t)=\mathcal{E}(t) u_{0}=\sum_{\ell=0}^{\infty} \sum_{k=1}^{N(n, \ell)} e^{-\lambda_{\ell} t} \widehat{\left(u_{0}\right)}{ }_{\ell, k} Y_{\ell k},
$$

that implies the smoothing property in the next theorem.

Theorem 4.6. Let $0 \leqslant \mathrm{q} \leqslant v$ and $\mathrm{m} \in\{0,1,2, \ldots\}$. If $\mathrm{u}_{0} \in \mathrm{H}^{\mathrm{s}}$ then $\mathcal{E}(\mathrm{t}) \mathrm{u}_{0} \in \mathrm{H}^{v}$ and

$$
\left\|\partial_{\mathrm{t}}^{\mathrm{m}} \mathcal{E}(\mathrm{t}) v\right\|_{\mathrm{H}^{v}} \leqslant \mathrm{C}_{\mathrm{T}} \mathrm{t}^{-(v-\mathrm{q}) / 2-\mathrm{m}}\|v\|_{\mathrm{H}^{\mathrm{q}}}, \quad \text { for } 0<\mathrm{t} \leqslant \mathrm{T} .
$$

Proof. Adapting the argument of Thomée [13, Lemma 3.2], we see from (4.9) that the generalized Fourier coefficients of $\partial_{t}^{m} \mathcal{E}(t) u_{0}$ are

$$
\left.\left\langle\partial_{t}^{m} \mathcal{E}(t) u_{0}, Y_{\ell k}\right\rangle=\left(-\lambda_{\ell}\right)^{m} e^{-\lambda_{\ell} t} \widehat{\left(u_{0}\right)}\right)_{\ell k}
$$

so by (2.3),

$$
\left.\left\|\partial_{t}^{m} \mathcal{E}(t) u_{0}\right\|_{H^{v}}^{2}=\sum_{\ell=0}^{\infty}\left(1+\lambda_{\ell}\right)^{v} \lambda_{\ell}^{2 m} e^{-2 \lambda_{\ell} t} \sum_{k=1}^{N(\ell, n)} \mid \widehat{\left(u_{0}\right)}\right)\left._{\ell k}\right|^{2} .
$$

The result follows because, with $s=\lambda_{\ell} t$,

$$
t^{v-q+2 m}\left(1+\lambda_{\ell}\right)^{v-q} \lambda_{\ell}^{2 m} e^{-2 \lambda_{\ell} t} \leqslant(T+s)^{v-q} s^{2 m} e^{-2 s} \leqslant C_{T} \quad \text { for } 0 \leqslant t \leqslant T .
$$


Let $\mathcal{T}: \mathrm{L}_{2} \rightarrow \mathrm{H}^{2}$ be the solution operator for the elliptic problem

$$
u-\triangle^{*} u=f \quad \text { on } \mathbb{S}^{n}
$$

that is, $\mathcal{T} f:=\mathfrak{u}$. Thus,

$$
\mathrm{a}_{1}(\mathcal{T} \mathfrak{f}, v)=\langle\mathfrak{f}, v\rangle \quad \text { for all } v \in \mathrm{H}^{1},
$$

and we can define $\mathcal{T}_{\mathrm{h}}: \mathrm{L}_{2} \rightarrow S_{\mathrm{h}}$ by

$$
a_{1}\left(\mathcal{T}_{h} f, \chi\right)=\langle f, \chi\rangle \quad \text { for all } \chi \in S_{h} .
$$

It follows that $\mathcal{T}_{h} f=R_{h} \mathfrak{u}=R_{h} \mathcal{T} f$ and $R_{h}=\mathcal{T}_{h}\left(I-\triangle^{*}\right)$. Since

$$
\left\langle\mathrm{f}, \mathcal{T}_{\mathrm{h}} w\right\rangle=\mathrm{a}_{1}\left(\mathcal{T}_{\mathrm{h}} \mathrm{f}, \mathcal{T}_{\mathrm{h}} w\right) \quad \text { for all } \mathrm{f}, \boldsymbol{v} \in \mathrm{L}_{2},
$$

we see that $\mathcal{T}_{h}$ is self-adjoint and (taking $\boldsymbol{w}=\mathrm{f}$ ) strictly positive-definite.

Rewriting the homogeneous equation (4.7) as $\partial_{\mathfrak{t}} \mathfrak{u}+\left(\mathrm{I}-\triangle^{*}\right) \mathfrak{u}=\mathfrak{u}$, we see that

$$
\mathcal{T} \partial_{t} \mathfrak{u}+\mathfrak{u}=\mathcal{T} \mathfrak{u} \quad \text { for } \mathfrak{t}>0, \quad \text { with } \mathfrak{u}(0)=\mathfrak{u}_{0},
$$

and similarly the corresponding semidiscrete problem (4.8) is equivalent to

$$
\mathcal{T}_{h} \partial_{t} u_{h}+\mathfrak{u}_{h}=\mathcal{T}_{h} u_{h} \quad \text { for } t>0, \quad \text { with } \mathfrak{u}_{h}(0)=\mathfrak{u}_{0 h} .
$$

Thus, the error $\mathrm{e}=\mathfrak{u}_{\mathfrak{h}}-\mathfrak{u}$ satisfies

$$
\mathcal{T}_{h} \partial_{t} \mathrm{e}+\mathrm{e}=\mathcal{T}_{h} \mathrm{e}+\rho \quad \text { where } \rho=\left(R_{h}-\mathrm{I}\right) \mathrm{u} .
$$

Lemma 4.7. With the notation above, if $\mathfrak{u}_{0 \mathrm{~h}}=\mathrm{P}_{\mathrm{h}} \mathfrak{u}_{0}$ then

$$
\|\mathrm{e}(\mathrm{t})\|^{2} \leqslant \mathrm{C}_{\mathrm{T}}\left(\|\rho(\mathrm{t})\|^{2}+\frac{1}{\mathrm{t}} \int_{0}^{\mathrm{t}}\left(\mathrm{s}^{2}\left\|\partial_{\mathrm{s}} \rho\right\|^{2}+\|\rho(\mathrm{s})\|^{2}\right) \mathrm{ds}\right) \quad \text { for } 0<\mathrm{t} \leqslant \mathrm{T} .
$$

Proof. We modify the argument of Thomée [13, Lemma 3.3]. Taking the inner product of (4.10) with $\partial_{\mathrm{t}} \mathrm{e}$ gives

$$
\left\langle\mathcal{T}_{h} \partial_{t} \mathrm{e}, \partial_{\mathrm{t}} \mathrm{e}\right\rangle+\left\langle\mathrm{e}, \partial_{\mathrm{t}} \mathrm{e}\right\rangle=\left\langle\mathcal{T}_{\mathrm{h}} \mathrm{e}+\rho, \partial_{\mathrm{t}} \mathrm{e}\right\rangle,
$$

and since $\left\langle\mathcal{T}_{h} \partial_{t} \mathrm{e}, \partial_{t} \mathrm{e}\right\rangle \geqslant 0$ and $\left\langle\mathrm{e}, \partial_{\mathrm{t}} \mathrm{e}\right\rangle=(1 / 2) \partial_{\mathrm{t}}\|\mathrm{e}\|^{2}$, it follows that

$$
\partial_{\mathrm{t}}\|\mathrm{e}\|^{2} \leqslant 2\left\langle\mathcal{T}_{\mathrm{h}} \mathrm{e}+\rho, \partial_{\mathrm{t}} \mathrm{e}\right\rangle,
$$


implying that

$$
\partial_{t}\left(t\|e\|^{2}\right)=\|e\|^{2}+t \partial_{t}\|e\|^{2} \leqslant\|e\|^{2}+2 t\left\langle\mathcal{T}_{h} e+\rho, \partial_{t} e\right\rangle .
$$

Since

$$
2 t\left\langle\mathcal{T}_{h} e, \partial_{t} e\right\rangle=t \partial_{t}\left\langle\mathcal{T}_{h} e, e\right\rangle \leqslant \partial_{t}\left(t\left\langle\mathcal{T}_{h} e, e\right\rangle\right)
$$

and

$$
\mathrm{t}\left\langle\rho, \partial_{\mathrm{t}} \mathrm{e}\right\rangle=\partial_{\mathrm{t}}(\mathrm{t}\langle\rho, \mathrm{e}\rangle)-\mathrm{t}\left\langle\partial_{\mathrm{t}} \rho, \mathrm{e}\right\rangle-\langle\rho, \mathrm{e}\rangle,
$$

we have

$$
\partial_{t}\left(t\|e\|^{2}\right) \leqslant\|e\|^{2}+\partial_{t}\left(t\left\langle\mathcal{T}_{h} \mathrm{e}+2 \rho, e\right\rangle\right)-2 t\left\langle\partial_{t} \rho, e\right\rangle-2\langle\rho, e\rangle,
$$

so integration gives

$$
\mathrm{t}\|\mathrm{e}\|^{2} \leqslant \int_{0}^{\mathrm{t}}\|\mathrm{e}(\mathrm{s})\|^{2} \mathrm{~d} s+\mathrm{t}\left\langle\mathcal{T}_{\mathrm{h}} \mathrm{e}+2 \rho, \mathrm{e}\right\rangle+2 \int_{0}^{\mathrm{t}}\left|\left\langle s \partial_{s} \rho+\rho(\mathrm{s}), \mathrm{e}(\mathrm{s})\right\rangle\right| \mathrm{d} s
$$

and using $2\langle\rho, \mathrm{e}\rangle \leqslant 4\|\rho\|^{2}+(1 / 2)\|\mathrm{e}\|^{2}$,

$$
\mathrm{t}\|\mathrm{e}\|^{2} \leqslant 2 \mathrm{t}\left\langle\mathcal{T}_{\mathrm{h}} \mathrm{e}, \mathrm{e}\right\rangle+8 \mathrm{t}\|\rho\|^{2}+2 \int_{0}^{\mathrm{t}}\left(\mathrm{s}^{2}\left\|\partial_{\mathrm{s}} \rho\right\|^{2}+\|\rho(\mathrm{s})\|^{2}+2\|\mathrm{e}(\mathrm{s})\|^{2}\right) \mathrm{d} s .
$$

To deal with the terms in e on the right-hand side, take the inner product of (4.10) with e, obtaining

$$
(1 / 2) \partial_{\mathrm{t}}\left\langle\mathcal{T}_{\mathrm{h}} \mathrm{e}, \mathrm{e}\right\rangle+\|\mathrm{e}\|^{2}=\left\langle\mathcal{T}_{\mathrm{h}} \mathrm{e}+\rho, \mathrm{e}\right\rangle,
$$

or equivalently, $\partial_{\mathrm{t}}\left\langle\mathcal{T}_{\mathrm{h}} \mathrm{e}, \mathrm{e}\right\rangle-2\left\langle\mathcal{T}_{\mathrm{h}} \mathrm{e}, \mathrm{e}\right\rangle+2\|\mathrm{e}\|^{2}=2\langle\rho, \mathrm{e}\rangle$. After multiplying by the integrating factor $e^{-2 t}$,

$$
\partial_{t}\left(e^{-2 t}\left\langle\mathcal{T}_{h} e, e\right\rangle\right)+2 e^{-2 t}\|e\|^{2}=2 e^{-2 t}\langle\rho, e\rangle,
$$

and the choice $\mathfrak{u}_{0 h}=P_{h} \mathfrak{u}_{0}$ means that $\mathcal{T}_{h} \mathrm{e}(0)=0$ because

$$
\left\langle\mathcal{T}_{h} e(0), w\right\rangle=\left\langle\mathcal{T}_{h}\left(P_{h}-I\right) u_{0}, w\right\rangle=\left\langle\left(P_{h}-I\right) u_{0}, \mathcal{T}_{h} w\right\rangle=0
$$

for every $w \in \mathrm{L}_{2}$. Thus,

$$
\begin{aligned}
e^{-2 t}\left\langle\mathcal{T}_{h} \mathrm{e}, \mathrm{e}\right\rangle+2 \int_{0}^{\mathrm{t}} \mathrm{e}^{-2 s}\|\mathrm{e}(\mathrm{s})\|^{2} \mathrm{~d} s=2 \int_{0}^{\mathrm{t}} \mathrm{e}^{-2 s}\langle\rho(\mathrm{s}), \mathrm{e}(\mathrm{s})\rangle \mathrm{d} s & \\
& \leqslant \int_{0}^{\mathrm{t}} e^{-2 s}\left(\|\rho(\mathrm{s})\|^{2}+\|\mathrm{e}(\mathrm{s})\|^{2}\right) \mathrm{ds},
\end{aligned}
$$


implying that

$$
\left\langle\mathcal{T}_{\mathrm{h}} \mathrm{e}, \mathrm{e}\right\rangle+\int_{0}^{\mathrm{t}} \mathrm{e}^{2(\mathrm{t}-\mathrm{s})}\|\mathrm{e}(\mathrm{s})\|^{2} \mathrm{~d} s \leqslant \int_{0}^{\mathrm{t}} e^{2(\mathrm{t}-\mathrm{s})}\|\rho(\mathrm{s})\|^{2} \mathrm{~d} s .
$$

Hence,

$$
2 t\left\langle\mathcal{T}_{\mathrm{h}} \mathrm{e}, \mathrm{e}\right\rangle+4 \int_{0}^{\mathrm{t}}\|\mathrm{e}(\mathrm{s})\|^{2} \mathrm{~d} s \leqslant 2 \max (\mathrm{t}, 2) \int_{0}^{\mathrm{t}} \mathrm{e}^{2(\mathrm{t}-\mathrm{s})}\|\rho(\mathrm{s})\|^{2} \mathrm{~d} s,
$$

and inserting this bound in (4.11) gives

$$
\|\mathrm{e}(\mathrm{t})\|^{2} \leqslant 8\|\rho(\mathrm{t})\|^{2}+\frac{2}{\mathrm{t}} \int_{0}^{\mathrm{t}} \mathrm{s}^{2}\left\|\partial_{\mathrm{s}} \rho\right\|^{2} \mathrm{~d} s+3 \max \left(1,2 \mathrm{t}^{-1}\right) \int_{0}^{\mathrm{t}} \mathrm{e}^{2(\mathrm{t}-\mathrm{s})}\|\rho(\mathrm{s})\|^{2} \mathrm{~d} s
$$

Theorem 4.8. Let $\mathrm{u}$ be the solution of the homogeneous problem (4.7) with initial data $\mathfrak{u}_{0}$, let $\mathfrak{u}_{\mathrm{h}}$ be the semidiscrete approximation given by (4.8) with $\mathfrak{u}_{0 \mathrm{~h}}=\mathrm{P}_{\mathrm{h}} \mathrm{u}_{0}$. For $1 \leqslant v \leqslant 2 \tau$ :

1. if $\mathrm{u}_{0} \in \mathrm{H}^{v}\left(\mathbb{S}^{n}\right)$, then

$$
\left\|u_{h}(t)-u(t)\right\| \leqslant C_{T} h_{X}^{v}\left\|u_{0}\right\|_{H^{v}} \text { for } 0 \leqslant t \leqslant T ;
$$

2. if $\mathrm{u}_{0} \in \mathrm{L}_{2}\left(\mathbb{S}^{n}\right)$ and $2 \tau$ is an integer, then

$$
\left\|u_{h}(t)-u(t)\right\| \leqslant C_{T} t^{-v / 2} h_{X}^{v}\left\|u_{0}\right\| \quad \text { for } 0<t \leqslant T .
$$

Proof. We see at once from Lemma 4.7 that

$$
\|e(t)\| \leqslant C_{t} \sup _{0 \leqslant s \leqslant t}\left(\|\rho(s)\|+s\left\|\partial_{s} \rho\right\|\right),
$$

and if $\mathrm{u}_{0} \in \mathrm{H}^{v}$ then, by Theorems 4.3 and 4.6 ,

$$
\|\rho(s)\|+s\left\|\partial_{s} \rho(s)\right\| \leqslant C h^{v}\left(\|u(s)\|_{H^{v}}+s\left\|\partial_{s} u(s)\right\|_{H^{v}}\right) \leqslant C h^{v}\left\|u_{0}\right\|_{H^{v}},
$$

which proves Part 1.

Assume now that $\mathfrak{u}_{0} \in \mathrm{L}_{2}$. By Theorems 4.3 and 4.6.

$$
\|\rho(t)\|=\left\|u(t)-R_{h} u(t)\right\| \leqslant C h\|u(t)\|_{H^{1}} \leqslant C h t^{-1 / 2}\left\|u_{0}\right\|,
$$


and the expansion (4.9) in spherical harmonics implies that

$$
\begin{aligned}
\int_{0}^{t}\|\rho(s)\|^{2} \mathrm{ds} & \leqslant \mathrm{Ch}^{2} \int_{0}^{\mathrm{t}}\|\mathrm{u}(\mathrm{s})\|_{\mathrm{H}^{1}}^{2} \mathrm{ds} \\
& =\mathrm{Ch}^{2} \sum_{\ell=0}^{\infty}\left(1+\lambda_{\ell}\right) \sum_{\mathrm{k}=1}^{\mathrm{N}(n, \ell)}\left|\widehat{\left(\mathrm{u}_{0}\right)_{\ell k}}\right|^{2} \int_{0}^{\mathrm{t}} \mathrm{e}^{-2 \lambda_{\ell} s} \mathrm{~d} s .
\end{aligned}
$$

If $\ell \geqslant 1$ then $\lambda_{\ell} \geqslant \lambda_{1}=\mathrm{n}$ so the substitution $s=\sigma / \lambda_{\ell}$ gives

$$
\left(1+\lambda_{\ell}\right) \int_{0}^{t} e^{-2 \lambda_{\ell} s} \mathrm{~d} s=\frac{1+\lambda_{\ell}}{\lambda_{\ell}} \int_{0}^{\lambda_{\ell} t} e^{-2 \sigma} d \sigma \leqslant\left(1+n^{-1}\right) \int_{0}^{\infty} e^{-2 \sigma} \mathrm{d} \sigma \leqslant 1,
$$

and thus

$$
\left.\int_{0}^{t}\|\rho(s)\|^{2} d s \leqslant\left. C^{2}\left(t \mid \widehat{(u}_{0}\right)_{01}\right|^{2}+\left.\sum_{\ell=1}^{\infty} \sum_{k=1}^{N(n, \ell)}\left|\widehat{\left(\mathfrak{u}_{0}\right)}\right|_{\ell k}\right|^{2}\right) \leqslant C_{t} h^{2}\left\|u_{0}\right\|^{2} .
$$

Similarly,

$$
\begin{aligned}
\int_{0}^{t} s^{2}\left\|\partial_{s} \rho\right\|^{2} d s & \leqslant \mathrm{Ch}^{2} \int_{0}^{t} s^{2}\left\|\partial_{s} u(s)\right\|_{H^{1}}^{2} d s \\
& =\mathrm{Ch}^{2} \sum_{\ell=1}^{\infty}\left(1+\lambda_{\ell}\right) \lambda_{\ell}^{2} \int_{0}^{t} s^{2} e^{-2 \lambda_{\ell} s} d s \sum_{k=1}^{N(n, \ell)} \mid \widehat{\left.\left(\mathfrak{u}_{0}\right)_{\ell k}\right|^{2}}
\end{aligned}
$$

and for all $\ell \geqslant 1$,

$$
\left(1+\lambda_{\ell}\right) \lambda_{\ell}^{2} \int_{0}^{t} s^{2} e^{-2 \lambda_{\ell} s} \mathrm{~d} s=\frac{1+\lambda_{\ell}}{\lambda_{\ell}} \int_{0}^{\lambda_{\ell} t} \sigma^{2} e^{-2 \sigma} \mathrm{d} \sigma \leqslant C,
$$

so $\int_{0}^{\mathrm{t}} \mathrm{s}^{2}\left\|\partial_{s} \rho\right\|^{2} \mathrm{ds} \leqslant \mathrm{Ch}^{2}\left\|\mathrm{u}_{0}\right\|^{2}$. Applying Lemma 4.7, Part 2 follows in the special case $v=1$.

To deal with case $v=2 \tau$, we introduce the solution operator for the semidiscrete problem, $\mathcal{E}_{\mathrm{h}}(\mathrm{t}) \mathfrak{u}_{0}:=\mathfrak{u}_{\mathrm{h}}(\mathrm{t})$, and use the semigroup property: $\mathcal{E}(s+t)=\mathcal{E}(s) \mathcal{E}(t)$ and $\mathcal{E}_{h}(s+t)=\mathcal{E}_{h}(s) \mathcal{E}_{h}(t)$ for all $s$ and $t$. The error operator $\mathcal{F}_{h}(t)=\mathcal{E}_{h}(t)-\mathcal{E}(t)$ satisfies the identity

$$
\begin{aligned}
\mathcal{F}_{h}(t)-\mathcal{F}_{h}(t / 2)^{2} & =\mathcal{E}_{h}(t / 2)^{2}-\mathcal{E}(t / 2)^{2}-\left[\mathcal{E}_{h}(t / 2)-\mathcal{E}(t / 2)\right]^{2} \\
& =\mathcal{F}_{h}(t / 2) \mathcal{E}(t / 2)+\mathcal{E}(t / 2) \mathcal{F}_{h}(t / 2),
\end{aligned}
$$


and by Part 1 and Theorem 4.6.

$$
\left\|\mathcal{F}_{h}(t / 2) \mathcal{E}(t / 2) u_{0}\right\| \leqslant C h^{v}\left\|\mathcal{E}(t / 2) u_{0}\right\|_{H^{v}} \leqslant C h^{v}(t / 2)^{-v / 2}\left\|u_{0}\right\|
$$

Since $\mathcal{E}(\mathrm{t} / 2)$ and $\mathcal{F}_{h}(\mathrm{t} / 2)$ are self-adjoint in $\mathrm{L}_{2}$, the same estimate holds for the reversed product $\mathcal{E}(\mathrm{t} / 2) \mathcal{F}_{\mathrm{h}}(\mathrm{t} / 2)$, and therefore

$$
\left\|\mathcal{F}_{h}(t) u_{0}\right\| \leqslant C t^{-v / 2} h^{v}\left\|u_{0}\right\|+C t^{-1 / 2} h\left\|\mathcal{F}_{h}(t / 2) u_{0}\right\|
$$

The stability estimates $\left\|\mathcal{E}(\mathbf{t}) \mathrm{u}_{0}\right\| \leqslant\left\|\mathrm{u}_{0}\right\|$ and $\left\|\mathcal{E}_{\mathrm{h}}(\mathrm{t}) \mathrm{u}_{0}\right\| \leqslant \mathrm{C}\left\|\mathrm{u}_{0}\right\|$ mean that it suffices to consider the case $t^{-1 / 2} h \leqslant 1$, when repeated application of the estimate (4.13) gives

$$
\left\|\mathcal{F}_{h}(t) u_{0}\right\| \leqslant C t^{-v / 2} h^{v}\left\|u_{0}\right\|+C\left(t^{-1 / 2} h\right)^{j}\left\|\mathcal{F}_{h}\left(t / 2^{j}\right) u_{0}\right\|
$$

for $j=0,1,2, \ldots, v=2 \tau$, and thus $\left\|\mathcal{F}_{h}(t) u_{0}\right\| \leqslant C t^{-\tau} h^{2 \tau}\left\|u_{0}\right\|$. For the remaining case $1<v<2 \tau$, let $\theta=v /(2 \tau)$ and observe that

$$
\begin{aligned}
\left\|\mathcal{F}_{h}(t) u_{0}\right\| & =\left\|\mathcal{F}_{h}(t) u_{0}\right\|^{1-\theta}\left\|\mathcal{F}_{h}(t) u_{0}\right\|^{\theta} \\
& \leqslant C\left\|u_{0}\right\|^{1-\theta}\left[\left(t^{-1 / 2} h\right)^{2 \tau}\left\|u_{0}\right\|\right]^{\theta}=C t^{-v / 2} h^{\nu}\left\|u_{0}\right\| .
\end{aligned}
$$

\section{$5 \quad$ Numerical experiments}

We present the results of some numerical experiments with two model problems. In both cases, the integration contour (3.4) and quadrature step $k$ are chosen as in Theorem 3.1, with

$$
\mathrm{T}=1, \quad \omega=1, \quad \theta=1 / 2, \quad \delta=\pi / 4, \quad \mathrm{r}=\pi / 4
$$

Figure 1] shows the case $\mathbf{N}=20$. Our conference paper [3] presents some earlier numerical examples.

\subsection{A scalar problem}

Consider the ODE $\mathfrak{u}^{\prime}+\mathfrak{u}=\mathbf{f}(\mathbf{t})$ for $\boldsymbol{t}>0$, with $\mathfrak{u}(0)=1$. We choose the source term $f$ so that the exact solution is

$$
u(t)=1+\frac{4 t^{3 / 2}}{3 \sqrt{\pi}}
$$




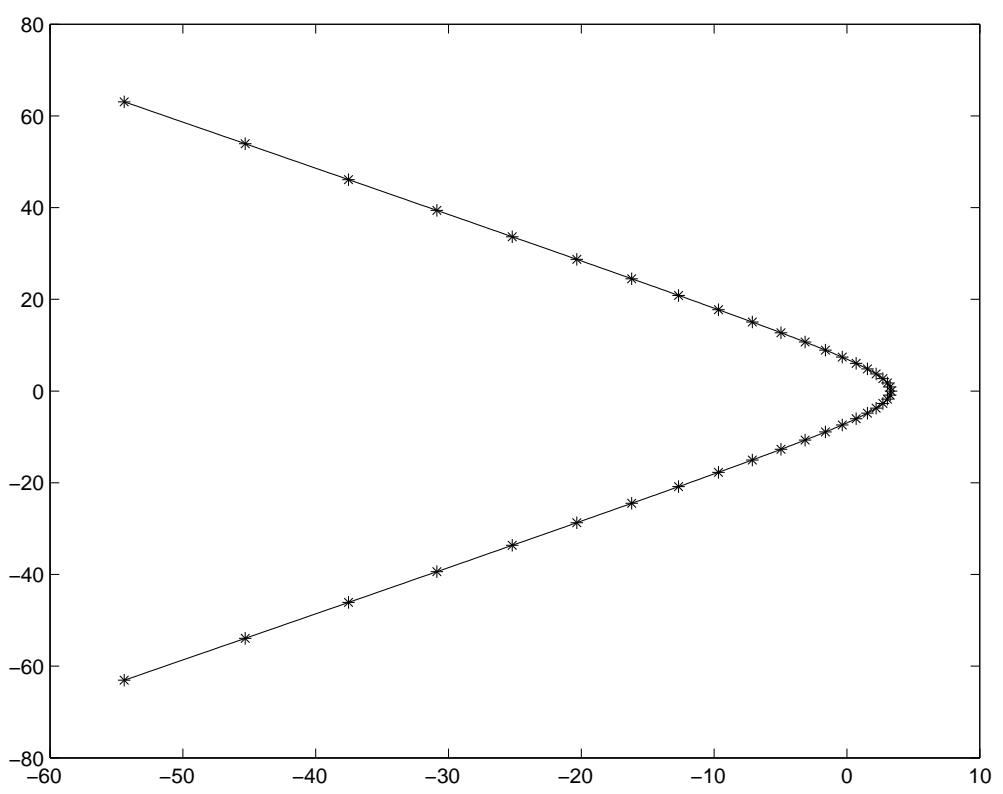

Figure 1: The integration contour $\Gamma$ and quadrature points $z_{\mathbf{j}}$ when $\mathbf{N}=20$.

which has the Laplace transform $\hat{\mathfrak{u}}(z)=z^{-1}+z^{-5 / 2}$. In this case, no spatial discretization is required, and the numerical solution $U_{N}$ is given by (3.8). Table 1 shows the error at $t=2$ for different values of $N$. The rapid convergence is consistent with the error bound of Theorem 3.1 , but as $\mathrm{N}$ increases the quadrature eventually becomes unstable.

\begin{tabular}{c|ccccc}
\hline $\mathrm{N}$ & 10 & 20 & 30 & 35 & 40 \\
\hline$\left|\mathrm{U}_{\mathrm{N}}(2)-\mathfrak{u}(2)\right|$ & $1.71 \mathrm{E}-04$ & $6.44 \mathrm{E}-08$ & $3.75 \mathrm{E}-11$ & $7.52 \mathrm{E}-13$ & $1.16 \mathrm{E}-12$ \\
\hline
\end{tabular}

Table 1: Errors for a scalar problem.

\subsection{Heat equation on the unit sphere}

Fix $0<a<1$ and define $u_{0}: \mathbb{S}^{2} \rightarrow \mathbb{C}$ for $x=\left(x_{1}, x_{2}, x_{3}\right) \in \mathbb{S}^{2}$ by

$$
u_{0}(x)= \begin{cases}1, & \text { if } a \leqslant x_{3} \leqslant 1 \\ 0, & \text { if }-1 \leqslant x_{3}<a\end{cases}
$$




\begin{tabular}{|c|c|c|c|}
\hline $\mathrm{m}$ & $\rho_{\mathrm{m}}(\mathrm{r})$ & Smoothness & $\tau$ \\
\hline 2 & $(1-r)_{+}^{6}\left(3+18 r+35 r^{2}\right)$ & $\mathrm{C}^{4}$ & $7 / 2$ \\
3 & $(1-\mathrm{r})_{+}^{8}\left(1+8 \mathrm{r}+25 \mathrm{r}^{2}+32 \mathrm{r}^{3}\right)$ & $\mathrm{C}^{6}$ & $9 / 2$ \\
\hline
\end{tabular}

Table 2: The compactly supported SRBFs of Wendland [16].

This axially symmetric function has the Fourier-Legendre expansion

$$
u_{0}(x)=\sum_{\ell=0}^{\infty} \widehat{\left(u_{0}\right)_{\ell}} P_{\ell}\left(x_{3}\right), \quad \text { where } \widehat{\left(u_{0}\right)_{\ell}}=\frac{2 \ell+1}{2} \int_{a}^{1} P_{\ell}(t) d t
$$

The zeroth coefficient is $\widehat{\left(\mathbf{u}_{0}\right)_{0}}=(1-\mathbf{a}) / 2$, and the remaining coefficients are expressible in terms of Jacobi polynomials [1, page 172], [7, Formula 18.9.15],

$$
\widehat{\left(u_{0}\right)_{\ell}}=\frac{1-a^{2}}{2} \frac{2 \ell+1}{\ell(\ell+1)} P_{\ell}^{\prime}(a)=\left(1-a^{2}\right) \frac{(2 \ell+1)}{4 \ell} P_{\ell-1}^{(1,1)}(a) \text { for } \ell \geqslant 1 \text {; }
$$

consequently $\widehat{\left(u_{0}\right)_{\ell}}=\mathrm{O}\left(\ell^{-1 / 2}\right)$ as $\ell \rightarrow \infty$ [12, Theorem 7.32.2].

The PDE $u_{t}-\triangle^{*} u=0$ with initial data (5.1) describes heat diffusion from a spherical cap about the north pole onto the surface of the unit sphere $\mathbb{S}^{2}$. By separating variables, we find that the exact solution is

$$
u(x, t)=\sum_{\ell=0}^{\infty} e^{-\ell(\ell+1) t} \widehat{\left(u_{0}\right)_{\ell}} P_{\ell}\left(x_{3}\right), \quad \text { for } x=\left(x_{1}, x_{2}, x_{3}\right) \in \mathbb{S}^{2} .
$$

For the spatial discretization, we use the compactly supported radial basis functions introduced by Wendland [16], for which the strictly positive-definite kernel has the form

$$
\Phi(x, y)=\rho_{m}(\sqrt{2-2 x \cdot y})
$$

In Table 2, we show $\rho_{2}$ and $\rho_{3}$ explicitly, along with the values of the exponent $\tau$ in (2.8). We generate the set of points $X$ using an equal area partitioning algorithm of Saff and Kuijlaars [9]. To compute the inner products arising in the matrix entries (3.15) and the load vector components $G_{p}(z)$, we use a quadrature approximation of the form

$$
\int_{\mathbb{S}^{2}} v \mathrm{~d} S \approx \frac{2 \pi}{\mathrm{R}} \sum_{\mathrm{q}=1}^{\mathrm{R}} \sum_{\mathrm{p}=1}^{\mathrm{R} / 2} w_{\mathrm{p}} v\left(\sin \theta_{\mathrm{p}} \cos \phi_{\mathrm{q}}, \sin \theta_{\mathrm{p}} \sin \phi_{\mathrm{q}}, \cos \theta_{\mathrm{p}}\right)
$$




\begin{tabular}{|c|c|c|c|c|c|c|}
\hline & $\mathrm{K}$ & 200 & 400 & 600 & 801 & 1001 \\
& $\mathrm{~h}_{\mathrm{X}}$ & 0.1796 & 0.1281 & 0.1039 & 0.0888 & 0.0794 \\
& $\mathrm{R}$ & 200 & 200 & 200 & 500 & 500 \\
\hline $\mathrm{N}=10$ & $e_{\max }$ & $5.67 \mathrm{E}-05$ & $5.06 \mathrm{E}-06$ & $1.67 \mathrm{E}-06$ & $9.89 \mathrm{E}-07$ & $8.24 \mathrm{E}-07$ \\
& $e_{2}$ & $4.63 \mathrm{E}-05$ & $3.60 \mathrm{E}-06$ & $1.10 \mathrm{E}-06$ & $8.23 \mathrm{E}-07$ & $7.81 \mathrm{E}-07$ \\
& $\mathrm{EOC}\left(\boldsymbol{e}_{2}\right)$ & & $7.56 \mathrm{E}+00$ & $5.66 \mathrm{E}+00$ & $1.84 \mathrm{E}+00$ & $4.66 \mathrm{E}-01$ \\
\hline $\mathrm{N}=20$ & $e_{\max }$ & $5.61 \mathrm{E}-05$ & $4.47 \mathrm{E}-06$ & $1.03 \mathrm{E}-06$ & $3.26 \mathrm{E}-07$ & $1.48 \mathrm{E}-07$ \\
& $\boldsymbol{e}_{2}$ & $4.61 \mathrm{E}-05$ & $3.53 \mathrm{E}-06$ & $8.07 \mathrm{E}-07$ & $2.64 \mathrm{E}-07$ & $1.20 \mathrm{E}-07$ \\
& $\mathrm{EOC}\left(\boldsymbol{e}_{2}\right)$ & & $7.60 \mathrm{E}+00$ & $7.05 \mathrm{E}+00$ & $7.11 \mathrm{E}+00$ & $7.06 \mathrm{E}+00$ \\
\hline $\mathrm{N}=30$ & $e_{\max }$ & $5.61 \mathrm{E}-05$ & $4.47 \mathrm{E}-06$ & $1.03 \mathrm{E}-06$ & $3.26 \mathrm{E}-07$ & $1.48 \mathrm{E}-07$ \\
& $\boldsymbol{e}_{2}$ & $4.61 \mathrm{E}-05$ & $3.53 \mathrm{E}-06$ & $8.07 \mathrm{E}-07$ & $2.64 \mathrm{E}-07$ & $1.20 \mathrm{E}-07$ \\
& $\mathrm{EOC}\left(\boldsymbol{e}_{2}\right)$ & & $7.60 \mathrm{E}+00$ & $7.05 \mathrm{E}+00$ & $7.11 \mathrm{E}+00$ & $7.06 \mathrm{E}+00$ \\
\hline $\mathrm{N}=35$ & $e_{\max }$ & $5.61 \mathrm{E}-05$ & $4.47 \mathrm{E}-06$ & $1.03 \mathrm{E}-06$ & $3.26 \mathrm{E}-07$ & $1.48 \mathrm{E}-07$ \\
& $e_{2}$ & $4.61 \mathrm{E}-05$ & $3.53 \mathrm{E}-06$ & $8.07 \mathrm{E}-07$ & $2.64 \mathrm{E}-07$ & $1.20 \mathrm{E}-07$ \\
& $\mathrm{EOC}\left(\boldsymbol{e}_{2}\right)$ & & $7.60 \mathrm{E}+00$ & $7.05 \mathrm{E}+00$ & $7.11 \mathrm{E}+00$ & $7.06 \mathrm{E}+00$ \\
\hline
\end{tabular}

Table 3: Numerical results with SRBFs constructed using $\rho_{2}$.

for an even number $R \geqslant 2$, where $\int_{-1}^{1} f(z) d z \approx \sum_{p=1}^{R / 2} w_{p} f\left(\cos \theta_{p}\right)$ is a GaussLegendre rule and $\phi_{\mathrm{q}}=2 \pi \mathrm{q} / \mathrm{R}$. The error in the approximation (5.2) is zero if the integrand $v$ is a polynomial of total degree $\mathrm{R}-1$ or less.

In the numerical experiments, we let $a=0.9$ in the definition (5.1) of $\mathfrak{u}_{0}$. Tables 3 and 4 show values of the quantities

$$
e_{\max }=\max _{x \in \mathcal{Q}}\left|u_{N, h}(x, 1)-u(x, 1)\right|
$$

and

$$
e_{2}=\left(\sum_{x \in \mathcal{Q}} w_{x}\left|u_{N, h}(x, 1)-u(x, 1)\right|^{2}\right)^{1 / 2},
$$

for different choices of $K$ and $R$. Here, $\mathcal{Q}$ is the set of quadrature points.

Since $u_{0} \in \mathrm{L}_{2}\left(\mathbb{S}^{2}\right)$, we expect from Theorem 4.8 and the triangle inequality (3.21) that if $N$ is sufficiently large then $e_{2}=O\left(h^{2 \tau}\right)$ - that is, $O\left(h^{7}\right)$ using $\rho_{2}$, and $\mathrm{O}\left(\mathrm{h}^{9}\right)$ using $\rho_{3}$. The observed convergence rates are close to these predicted values. We remark that when $\mathrm{K}=1001$, the condition number of the linear system (3.19) is around $10^{7}$ using $\rho_{2}$, and around $10^{9}$ using $\rho_{3}$, so we cannot expect to reduce the error much below the smallest values shown in the tables. 


\begin{tabular}{|c|c|c|c|c|c|c|}
\hline & $\mathrm{K}$ & 200 & 400 & 600 & 801 & 1001 \\
& $\mathrm{~h}_{\mathrm{X}}$ & 0.1796 & 0.1281 & 0.1039 & 0.0888 & 0.0794 \\
& $\mathrm{R}$ & 200 & 200 & 200 & 500 & 500 \\
\hline $\mathrm{N}=10$ & $\boldsymbol{e}_{\max }$ & $6.86 \mathrm{E}-05$ & $3.84 \mathrm{E}-06$ & $1.17 \mathrm{E}-06$ & $8.35 \mathrm{E}-07$ & $7.79 \mathrm{E}-07$ \\
& $\boldsymbol{e}_{2}$ & $3.93 \mathrm{E}-05$ & $1.63 \mathrm{E}-06$ & $7.85 \mathrm{E}-07$ & $7.74 \mathrm{E}-07$ & $7.71 \mathrm{E}-07$ \\
& $\mathrm{EOC}\left(\boldsymbol{e}_{2}\right)$ & & $9.41 \mathrm{E}+00$ & $3.50 \mathrm{E}+00$ & $9.13 \mathrm{E}-02$ & $3.37 \mathrm{E}-02$ \\
\hline $\mathrm{N}=20$ & $e_{\max }$ & $6.78 \mathrm{E}-05$ & $3.11 \mathrm{E}-06$ & $4.54 \mathrm{E}-07$ & $8.98 \mathrm{E}-08$ & $3.24 \mathrm{E}-08$ \\
& $\boldsymbol{e}_{2}$ & $3.91 \mathrm{E}-05$ & $1.45 \mathrm{E}-06$ & $2.12 \mathrm{E}-07$ & $4.83 \mathrm{E}-08$ & $1.73 \mathrm{E}-08$ \\
& $\mathrm{EOC}\left(\boldsymbol{e}_{2}\right)$ & & $9.75 \mathrm{E}+00$ & $9.17 \mathrm{E}+00$ & $9.41 \mathrm{E}+00$ & $9.18 \mathrm{E}+00$ \\
\hline $\mathrm{N}=30$ & $e_{\max }$ & $6.78 \mathrm{E}-05$ & $3.11 \mathrm{E}-06$ & $4.54 \mathrm{E}-07$ & $8.98 \mathrm{E}-08$ & $3.24 \mathrm{E}-08$ \\
& $\boldsymbol{e}_{2}$ & $3.91 \mathrm{E}-05$ & $1.45 \mathrm{E}-06$ & $2.12 \mathrm{E}-07$ & $4.83 \mathrm{E}-08$ & $1.73 \mathrm{E}-08$ \\
& $\mathrm{EOC}\left(\boldsymbol{e}_{2}\right)$ & & $9.75 \mathrm{E}+00$ & $9.17 \mathrm{E}+00$ & $9.41 \mathrm{E}+00$ & $9.18 \mathrm{E}+00$ \\
\hline $\mathrm{N}=35$ & $e_{\max }$ & $6.78 \mathrm{E}-05$ & $3.11 \mathrm{E}-06$ & $4.54 \mathrm{E}-07$ & $8.98 \mathrm{E}-08$ & $3.24 \mathrm{E}-08$ \\
& $\boldsymbol{e}_{2}$ & $3.91 \mathrm{E}-05$ & $1.45 \mathrm{E}-06$ & $2.12 \mathrm{E}-07$ & $4.83 \mathrm{E}-08$ & $1.73 \mathrm{E}-08$ \\
& $\mathrm{EOC}\left(\boldsymbol{e}_{2}\right)$ & & $9.75 \mathrm{E}+00$ & $9.17 \mathrm{E}+00$ & $9.41 \mathrm{E}+00$ & $9.18 \mathrm{E}+00$ \\
\hline
\end{tabular}

Table 4: Numerical results with SRBFs constructed using $\rho_{3}$.

Acknowledgement The first author is supported by the Australian Research Council.

\section{References}

[1] W. E. Bailey. An Elementary Treatise on Fourier's Series, and Spherical, Cylindrical, and Ellipsoidal Harmonics, with Applications to Problems in Mathematical Physics. Dover, New York, 1959.

[2] D. Chen, V. A. Menegatto, and X. Sun. A necessary and sufficient condition for strictly positive definite functions on spheres. Proc. Amer. Math. Soc., 131:2733-2740, 2003.

[3] Quoc Thong Le Gia and William McLean. Numerical solution of a parabolic equation on the sphere using Laplace transforms and radial basis functions. In W. McLean and A. J. Roberts, editors, Proceedings of the 15th Biennial Computational Techniques and Applications Conference, CTAC-2010, volume 52 of ANZIAM J., pages C89-C102, 2011. 
[4] Q. T. Le Gia. Approximation of parabolic PDEs on spheres using spherical basis functions. Adv. Comput. Math., 22:377-397, 2005.

[5] W. McLean and V. Thomée. Numerical solution via Laplace transforms of a fractional order evolution equation. J. of Integral Equations and Applications, 22:57-94, 2010.

[6] C. Müller. Spherical Harmonics, volume 17 of Lecture Notes in Mathematics. Springer-Verlag, Berlin, 1966.

[7] National Institute of Standards and Technology. Digital Library of Mathematical Functions. Release date 2011-07-01, http://dlmf.nist.gov/.

[8] J.A. Nitsche and A.H. Schatz. Interior estimates for Ritz-Galerkin methods. Math. Comp., 28:937-958, 1974.

[9] E. B. Saff and A. B. J. Kuijlaars. Distributing many points on a sphere. Math. Intelligencer, 19:5-11, 1997.

[10] I. J. Schoenberg. Positive definite function on spheres. Duke Math. J., 9:96-108, 1942.

[11] D. Sheen, I. H .Sloan, and V .Thomée. A parallel method for timediscretization of parabolic equations based on contour integral representation and quadrature. Math. Comp., 69:177-195, 1999.

[12] G. Szegö. Orthogonal Polynomials. American Mathematical Society, New York, 1959.

[13] Vidar Thomée. Galerkin Finite Element Methods for Parabolic Problems. Springer, Berlin, 1997.

[14] T. Tran, Q. T. Le Gia, I. H. Sloan, and E. P. Stephan. Boundary integral equations on the sphere with radial basis functions: error analysis. Appl. Numer. Math., 59:2857-2871, 2009.

[15] T. Tran and T. D. Pham. Pseudodifferential equations on the sphere with spherical radial basis functions: Error analysis. Applied Mathematics Report 2008/11, The University of New South Wales, 2008.

[16] H. Wendland. Scattered Data Approximation. Cambridge University Press, Cambridge, 2005. 
[17] Y. Xu and E. W. Cheney. Strictly positive definite functions on spheres. Proc. Amer. Math. Soc., 116:977-981, 1992. 